\title{
Clinicopathological Progress of Colorectal Resection in Gynecological Malignancy Patients : Does the risk of anastomotic leakage increase after surgery?
}

\section{Ayoung Kang}

Pusan National University Yangsan Hospital, Pusan National University School of Medicine

\section{Byung-Soo Park}

Pusan National University Yangsan Hospital, Pusan National University School of Medicine

\section{Sung Hwan Cho}

Pusan National University Yangsan Hospital, Pusan National University School of Medicine

\section{Gyung Mo Son}

Pusan National University Yangsan Hospital, Pusan National University School of Medicine

\section{Yong Jung Song}

Pusan National University Yangsan Hospital, Pusan National University School of Medicine Hyun Sung Kim ( $\square$ gustohs@gmail.com )

Pusan National University Yangsan Hospital, Pusan National University School of Medicine

\section{Research Article}

Keywords: Colorectal resection, Gynecological malignancy, Anastomotic leakage, Cooperative surgery

Posted Date: September 2nd, 2021

DOl: https://doi.org/10.21203/rs.3.rs-737626/v1

License: (c) (i) This work is licensed under a Creative Commons Attribution 4.0 International License.

Read Full License 


\section{Abstract}

Objective: This study was conducted to investigate the clinicopathological outcomes of colorectal resection in patients with advanced gynecological malignancies.

Methods: We reviewed the medical records of 104 patients of gynecological cancer with co-operation for colorectal resection from December 2008 to August 2020 by retrospective cohort study in a single center (PNUYH). Variables for risk factors and postoperative complications were compared by descriptive statistics. We excluded the cases with malignancies from other than female genital organs, gynecological benign diseases, primary creation of stoma, and any other bowel surgeries except colorectal resection.

Results: Of 104 patients in total, the mean age was 62.0 years. The most common gynecological malignancy was ovarian cancer( 85 patients, $81.7 \%$ ) and the most commonly performed operation was low anterior resection(80 patients, $76.9 \%) .61$ patients(58.7\%) had postoperative complications, while only 3 patients $(2.9 \%)$ with anastomotic leakage. Only preoperative albumin was statistically significant $(p=0.019)$ among the risk factors.

Conclusion: Our experience suggests that colorectal resection can be safely and efficaciously performed in patients with advanced gynecological malignancy.

\section{Background}

Gynecological malignancies such as cervical cancer, ovarian cancer, and endometrial cancer often invade adjacent organs at advanced stages, and in particular, colonic, and rectal invasions are frequent [1-3]. In most gynecological malignancies, curative surgical resection is the key to treatment and survival rate. Advanced gynecological malignancies often accompany intraabdominal metastasis, and sometimes induce intestinal obstruction too so that colectomy and proctectomy are required. Therefore, surgical collaboration is often required for colonic and rectal resections during gynecological malignancy surgery, and in cases where cooperative surgery has been performed, more careful post-operative management is required, and regular follow-up is important.

Patients who underwent colectomy may experience various complications during the recovery process. The range of complications is wide, from general ones that may occur after surgery performed under general anesthesia, to those caused by gastrointestinal surgery due to conditions such as intestinal obstruction and gastrointestinal tract bleeding, enteritis, and anastomotic leakage. The main goal of postoperative management of surgical patients is the recovery processes and the prevention and treatment of complications as such. However, there are not many studies on the progress after cooperative colorectal resection was performed in gynecological malignancy patients, and most of these studies were conducted from the perspective of obstetrics and gynecology.

In this paper, cooperative colorectal resection for gynecological malignancies performed at a single institution for 12 years was studied. This study aims to find a way to prevent postoperative complications 
and improve the survival rate by examining the progress after performing colorectal resection in gynecological malignancy patients from the viewpoint of the colorectal surgeon.

\section{li. Methods}

This study was conducted as a retrospective cohort study in a single institution. A database of patients who underwent cooperative surgery due to obstetrics and gynecological diseases at Pusan National University Yangsan Hospital between December 2008 and August 2020 was obtained, and through database classification and medical record analysis, patients who underwent colorectal resection due to gynecological malignancies were finally selected as the subjects. Cases of malignancies that originated from other organs than female reproductive organs (Krukenberg tumor, metastasis of colon cancer, etc.) or benign gynecological diseases (endometriosis, female pelvic inflammatory disease, rectovaginal fistula, etc.), cases where any kind of enterostomy was performed at the time of the first surgery, cases where only enterectomy was performed excluding the colon and the rectum (appendectomy only, small intestine resection, etc.), and cases where there was no anastomotic site because only primary repair was performed were excluded (Fig. 1).

Among the medical records collected, the records of the first medical examination at the department of obstetrics and gynecology or surgery, progress records, consultation request forms, operative notes, preanesthesia evaluation tables, anesthesia records, and hospitalized nursing records were analyzed. Examination results, histopathologic examination results, and imagery interpretation reports were also included. Referring to previous studies, observation items and variables necessary to analyze risk factors were selected [1, 4-6]. As patient factors, age, height, weight, body mass index (BMI), whether diabetic, whether smoking, steroid administration history, and American Society of Anesthesiology (ASA) classifications were included. Diagnosis and disease stages were determined according to the standards of the International Federation of Gynecology and Obstetrics (FIGO). Preoperative and postoperative hemoglobin $(\mathrm{Hb})$ levels, preoperative albumin (Alb) levels, and preoperative levels of CA125, CA19-9, and SCC, which are the most widely used tumor markers in gynecological malignancies, were investigated. The most recent examination results before entering the operating room were collected as preoperative examination results, and the results of the examination conducted for the first time after surgery were collected as postoperative examination results. As preoperative status-related factors, whether the relevant disease was diagnosed for the first time or had recurred, and whether chemotherapy and radiation therapy were carried out before surgery were included, and as intraoperative status-related factors, the total operation time, estimated blood loss (EBL), whether blood was transfused, and transfusion volume were included. As surgery-related factors, whether the surgery was emergency surgery, whether laparotomy was performed, types of surgery, whether an additional enterectomy accompanied, the distance from the anal verge (AV) to the anastomotic site, and anastomosis method (whether an automatic stapler was used) were investigated. In addition, whether the obstetrics and gynecology department requested cooperation from the department of surgery before the surgery was investigated and included in the variables. 
Whether complications occurred after surgery was investigated to find out the frequencies of surgical wound infection, anastomotic leakage, anastomotic site infection, intraabdominal infection, cardiovascular complications, respiratory complications, and urinary complications separately. Whether anastomotic leakage occurred was judged by putting together medical records and examination results using the diagnostic criterion set as corresponding to at least one of 1) cases where amylase and lipase were detected in the intraabdominal drainage tube contents in amounts exceeding the reference values, 2) cases where findings indicating anastomotic leakage such as fluid retention around the anastomotic site were identified in radiological findings such as computed tomography (CT) [5, 7]. Cases that did not meet this diagnostic criterion were classified as anastomotic site infection, and intraabdominal inflammations and infection of areas other than anastomotic sites were separately classified as intraabdominal infection. In order to explore and analyze risk factors for anastomotic leakage, the study subjects were divided into two groups: patients in whom anastomotic leakage occurred and patients in whom no anastomotic leakage occurred, and statistical analysis was attempted.

All statistical analyses were conducted using IBM SPSS Statistics version 25 (SPSS Inc, Chicago, IL, USA). Some scale variables were converted into nominal variables through grouping and analyzed thereafter. Chi-square tests and Fisher's exact tests were used for nominal variables and Mann-Whitney's $\mathrm{U}$ tests were used for scale variables. The P-value to determine statistical significance was set to below 0.05 .

This study is a retrospective cohort study conducted through medical record analysis and was exempted from review by the Institutional Review Board (IRB) of Pusan National University Yangsan Hospital. (IRB No. 05-2020-203). Written informed consent forms concerning cooperative colorectal resection were obtained for publication of the study.

\section{lii. Results}

According to the inclusion and exclusion criteria, 104 patients were finally selected as study subjects. Due to the characteristic of the patient group having gynecological malignancies, all patients were adult women.

The mean age of the study subjects was 62.0 years. Of the study subjects, 66 (63.5\%) were younger than 65 years and $38(36.5 \%)$ were 65 years old or older. The mean BMI was 22.9 , and 35 patients $(33.7 \%)$ had a BMI not lower than 24.0, which is commonly used as the standard for obesity in Korea. As for ASA classifications, 42 patients (40.4\%) were class I, 49 patients (47.1\%) were class II, 13 patients $(12.5 \%)$ were class III, and there were no patients corresponding to class IV or V. In the classifications by diagnosis, ovarian cancer accounted for the highest ratio with 85 patients $(81.7 \%)$, followed by cervical cancer (six patients), endometrial cancer (five patients), peritoneal cancer (four patients), uterine sarcoma (two patients), fallopian tubal cancer (one patient), and other cancer (one patient, malignant mixed Mullerian tumor (MMMT)). Among the study subjects, one patient was diagnosed with cervical cancer and endometrial cancer simultaneously and one patient was diagnosed with endometrial cancer and 
ovarian cancer simultaneously. These patients corresponded to double primary cancer cases in which two primary cancers were simultaneously diagnosed. In the database used for statistical analysis, these patients were classified based on the diagnosis with the higher stage between the two diagnoses.

Anticancer treatment including preoperative concurrent chemo-radiotherapy (CCRT) was performed in a total of 16 patients and radiation therapy was performed in a total of four patients (Table 1). 
Table 1

Demographics of patient characteristics $(n=104)$

\section{Factors}

Age (years)

$<65$

$\geq 65$

$\mathrm{BMI}$

$<24.0$

$\geq 24.0$

ASA

I

II

III

IV

$\mathrm{V}$

Diagnosis*

Cervical cancer

Endometrial cancer

Uterine sarcoma

Ovarian cancer

Fallopian tubal cancer

Peritoneal cancer

Others**
$31-90(62.0)$

66 (63.5\%)

38 (36.5\%)

16.5-30.8 (22.9)

$69(66.3 \%)$

35 (33.7\%)

$42(40.4 \%)$

$49(47.1 \%)$

$13(12.5 \%)$

0

0 


\section{Factors}

Preoperative chemotherapy

No $88(84.6 \%)$

Preoperative neoadjuvant $4(3.8 \%)$

Preoperative CCRT $3(2.9 \%)$

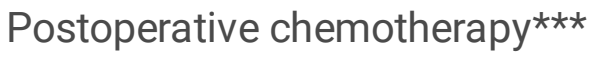
$9(8.7 \%)$

Preoperative radiotherapy

No $100(96.2 \%)$

Preoperative CCRT $3(2.9 \%)$

Preoperative radiotherapy only $1(1.0 \%)$

* 2 patients with double primary cancer were classified as the diseases with higher stage.

** Malignant mixed Mullerian tumors (MMMT)

*** Adjuvant chemotherapy after the first operation for gynecological malignancies in patients with colorectal resection for recurred gynecological malignancies

* Data is presented as min - max (mean) or number (\%).

Among colorectal resections, the most frequently performed operation was low anterior resection (LAR), which was performed in a total of 80 patients $(76.9 \%)$ followed by anterior resection (AR) performed in 10 patients (9.6\%), total colectomy (TC) (six patients, 5.8\%), and total proctocolectomy (TPC) (five patients, $4.8 \%$ ), and right hemicolectomy ( $\mathrm{RHC}$ ) (three patients, $2.9 \%)$. Other combined gastrointestinal resections in addition to the main operation were performed in 25 patients. Cases where only appendectomy was accompanied were the most frequent (10 patients, 9.6\%), followed by small intestine resection (eight patients, $7.7 \%$ ) and colectomy (seven patients, 6.7\%). Among the 104 patients, 103 (99.0\%) patients underwent laparotomy before surgery. In the case of the one remaining patient, surgery began with diagnostic laparoscopy but was switched to open surgery after the obstetrics and gynecology department identified the surgical findings and the actual surgical operation began as open surgery. Therefore, it is reasonable to assume that all 104 subjects underwent open surgery. Anastomosis using an automatic stapler was performed in 102 patients (98.1\%), and hand-sewn anastomosis was performed in two patients (1.9\%). The mean operation time was 402.2 minutes, ranging from a minimum of 60 minutes to a maximum of 935 minutes (Table 2). 
Table 2

Operation characteristics of colorectal resection

\section{Operation}

Low anterior resection

Anterior resection

Total colectomy

Total proctocolectomy

Right hemicolectomy

Additional bowel resection

No

Colectomy

Small intestine resection

Appendectomy

Laparotomy or laparoscopy

Laparotomy

$103(99.0 \%)$

Laparoscopy

Laparoscopy converted to open laparotomy*

Anastomosis

Stapler

Hand-sewn

Operation time (min)
80 (76.9\%)

10 (9.6\%)

$6(5.8 \%)$

5 (4.8\%)

$3(2.9 \%)$

79 (76.0\%)

$7(6.7 \%)$

$8(7.7 \%)$

$10(9.6 \%)$

* Began as diagnostic laparoscopy by gynecologist and switched to open laparotomy before the start of co-operation with general surgeon

Postoperative complications occurred in 61 (58.7\%) of the 104 patients. Among them, three cases (2.9\%) were clinically confirmed as anastomotic leakage. All three patients underwent reoperation immediately upon confirmation of leakage, and they improved and were discharged normally, although there were differences in their recovery periods. Three cases (2.9\%) of anastomotic site infection excluding leakage, and six cases $(5.8 \%)$ of intraabdominal infection other than anastomotic sites were identified. In addition, there were wound infections in eight patients $(7.7 \%)$, gastrointestinal complications such as enteroplegia and intestinal obstruction in 18 patients (17.3\%), cardiovascular complications in seven patients (6.7\%), respiratory system complications in five patients (4.8\%), and urinary system complications in 16 patients (15.4\%). Other complications identified were lymphedema in two patients, obturator nerve damage in one 
patient, poor blood sugar control in one patient, and multi-organ failure in one patient. There was no case of death as a direct result of postoperative complications except for one patient with multiple organ failure (Table 3).

Table 3

Postoperative mortality and morbidity

\begin{tabular}{|ll|}
\hline Postoperative mortality & $\mathbf{0}(\mathbf{0} \%)$ \\
\hline Postoperative morbidity & $61(58.7 \%)$ \\
Surgical wound infection & $8(7.7 \%)$ \\
Anastomotic leakage & $3(2.9 \%)$ \\
Anastomotic site infection (except leakage) & $3(2.9 \%)$ \\
Gastraabdominal infection (except anastomosis site) & $6(5.8 \%)$ \\
Cardiovascular complications & $18(17.3 \%)$ \\
Respiratory complications & $7(6.7 \%)$ \\
Urinary complications & $5(4.8 \%)$ \\
Others & $16(15.4 \%)$ \\
\hline * Multiple complications in one patient were counted respectively \\
\hline
\end{tabular}

The data on the comparison of individual characteristics according to whether there was anastomotic leakage were organized in Table 4. Analysis was conducted to identify risk factors for anastomotic leakage. Among the risk factors, the preoperative albumin level $(p=0.019)$ was the only risk factor that showed a statistically significant difference, and none of the remaining risk factors showed statistical significance. 
Table 4

Comparison between patients with and without anastomotic leakage

\begin{tabular}{|c|c|c|c|c|}
\hline & Total $(n=104)$ & $\begin{array}{l}\text { No leakage }(n= \\
101)\end{array}$ & $\begin{array}{l}\text { With leakage ( } \\
=3 \text { ) }\end{array}$ & $\begin{array}{l}\mathrm{P} \text { - } \\
\text { value }\end{array}$ \\
\hline Age (years) & $62.0(31-90)$ & $62.0(31-90)$ & $64.7(50-78)$ & 0.301 \\
\hline$<65$ & $66(63.5 \%)$ & $65(64.4 \%)$ & $1(33.3 \%)$ & \\
\hline$\geq 65$ & 38 (36.5\%) & $36(35.6 \%)$ & $2(66.7 \%)$ & \\
\hline Height (cm) & $\begin{array}{l}156.1(141.2- \\
169.2)\end{array}$ & $\begin{array}{l}156.0(141.2- \\
169.2)\end{array}$ & $\begin{array}{l}160.3(156.4- \\
163.4)\end{array}$ & 0.197 \\
\hline Weight (kg) & $\begin{array}{l}55.80(40.00- \\
72.30)\end{array}$ & $\begin{array}{l}55.89(40.00- \\
72.30)\end{array}$ & $\begin{array}{l}53.00(49.00- \\
57.98)\end{array}$ & 0.433 \\
\hline \multirow{3}{*}{$\begin{array}{l}\text { BMI } \\
<24.0 \\
\geq 24.0\end{array}$} & $\begin{array}{l}22.90(16.47- \\
30.78)\end{array}$ & $\begin{array}{l}22.97(16.47- \\
30.78)\end{array}$ & $\begin{array}{l}20.71(18.35- \\
23.70)\end{array}$ & 0.288 \\
\hline & 69 (66.3\%) & 66 (65.3\%) & $3(100.0 \%)$ & \\
\hline & 35 (33.7\%) & 35 (34.7\%) & 0 & \\
\hline \multicolumn{5}{|l|}{ ASA } \\
\hline I & $42(40.4 \%)$ & $41(40.6 \%)$ & $1(33.3 \%)$ & 0.915 \\
\hline II & 49 (47.1\%) & 49 (48.5\%) & 0 & \\
\hline III & $13(12.5 \%)$ & $11(10.9 \%)$ & $2(66.7 \%)$ & \\
\hline IV & 0 & 0 & 0 & \\
\hline V & 0 & 0 & 0 & \\
\hline \multicolumn{5}{|l|}{ DM } \\
\hline No & 95 (91.3\%) & 92 (91.1\%) & $3(100.0 \%)$ & 0.760 \\
\hline Yes & $9(8.7 \%)$ & $9(8.9 \%)$ & 0 & \\
\hline \multicolumn{5}{|l|}{ Smoking } \\
\hline No & $101(97.1 \%)$ & 99 (98.0\%) & $2(66.7 \%)$ & 0.085 \\
\hline Yes & $3(2.9 \%)$ & $2(2.0 \%)$ & 1 (33.3\%) & \\
\hline \multicolumn{5}{|c|}{ Steroid therapy } \\
\hline No & 101 (97.1\%) & 98 (97.0\%) & $3(100.0 \%)$ & 0.915 \\
\hline Yes & $3(2.9 \%)$ & $3(3.0 \%)$ & $0(0.0 \%)$ & \\
\hline
\end{tabular}




\begin{tabular}{|c|c|c|c|c|}
\hline & Total $(n=104)$ & $\begin{array}{l}\text { No leakage }(n= \\
101)\end{array}$ & $\begin{array}{l}\text { With leakage ( } \mathrm{n} \\
=3 \text { ) }\end{array}$ & $\begin{array}{l}\mathrm{P}- \\
\text { value }\end{array}$ \\
\hline Preop Alb (g/dL) & $3.83(2.0-4.7)$ & $3.86(2.0-4.7)$ & $2.80(2.1-3.8)$ & 0.019 \\
\hline$<3.0$ & $9(8.7 \%)$ & $7(6.9 \%)$ & $2(66.7 \%)$ & \\
\hline$\geq 3.0$ & $95(91.3 \%)$ & $94(93.1 \%)$ & $1(33.3 \%)$ & \\
\hline Preop Hb (g/dL) & $11.95(7.0-15.2)$ & $11.92(7.0-15.2)$ & $\begin{array}{l}12.87(11.6- \\
14.9)\end{array}$ & 0.456 \\
\hline Postop Hb (g/dL) & $11.60(6.0-16.5)$ & $11.55(6.0-16.5)$ & $\begin{array}{l}13.70(11.6- \\
15.1)\end{array}$ & 0.078 \\
\hline Operation time (min) & $402.2(60-935)$ & $400.8(60-935)$ & $446.7(330-540)$ & 0.422 \\
\hline Emergency operation & 93 (89.4\%) & $90(89.1 \%)$ & $3(100 \%)$ & 0.713 \\
\hline Elective & $11(10.6 \%)$ & $11(10.9 \%)$ & 0 & \\
\hline \multicolumn{5}{|l|}{ Emergency } \\
\hline \multicolumn{5}{|l|}{ Consult } \\
\hline Outpatient & $1(1.0 \%)$ & $1(1.0 \%)$ & 0 & 0.984 \\
\hline Preoperative & $33(31.7 \%)$ & $32(31.7 \%)$ & $1(33.3 \%)$ & \\
\hline Postoperative & 0 & 0 & 0 & \\
\hline No & 70 (67.3\%) & $68(67.3 \%)$ & $2(66.7 \%)$ & \\
\hline $\mathrm{EBL}(\mathrm{cc})$ & $\begin{array}{l}1239.4(100- \\
5000)\end{array}$ & $\begin{array}{l}1236.60(100- \\
5000)\end{array}$ & $\begin{array}{l}1333.33(200- \\
2300)\end{array}$ & 0.817 \\
\hline \multicolumn{5}{|l|}{ Intraop transfusion } \\
\hline No & $29(27.9 \%)$ & $29(28.7 \%)$ & 0 & 0.371 \\
\hline Yes & $75(72.1 \%)$ & $72(71.3 \%)$ & $3(100.0 \%)$ & \\
\hline \multicolumn{5}{|l|}{ Transfusion (pint) } \\
\hline RBC & $2.5(0-29)$ & $2.4(0-29)$ & $3.7(1-5)$ & 0.226 \\
\hline FFP & $0.9(0-29)$ & $0.9(0-29)$ & $1.22(0-3.6)$ & 0.686 \\
\hline \multicolumn{5}{|l|}{ Laparotomy or laparoscopy } \\
\hline Laparotomy & $103(99.0 \%)$ & $100(99.0 \%)$ & $3(100.0 \%)$ & 0.971 \\
\hline Laparoscopy & 0 & 0 & 0 & \\
\hline $\begin{array}{l}\text { Laparoscopy converted to } \\
\text { laparotomy }\end{array}$ & $1(1.0 \%)$ & $1(1.0 \%)$ & 0 & \\
\hline
\end{tabular}




\section{$\begin{array}{llll}\text { Total }(n=104) & \left.\begin{array}{l}\text { No leakage }(n= \\ 101\end{array}\right) & \left.\begin{array}{l}\text { With leakage }(n \\ =3\end{array}\right) & \begin{array}{l}\text { P- } \\ \text { value }\end{array}\end{array}$}

\section{Diagnosis}

Cervical cancer

$6(5.8 \%)$

$6(5.9 \%)$

0

0.756

Endometrial cancer

$5(4.8 \%)$

$5(5.0 \%)$

0

Uterine sarcoma

$2(1.9 \%)$

$2(2.0 \%)$

0

Ovarian cancer

$85(81.7 \%)$

$82(81.2 \%)$

$3(100 \%)$

Fallopian tubal cancer

$1(1.0 \%)$

$1(1.0 \%)$

0

Peritoneal cancer

$4(3.8 \%)$

$4(4.0 \%)$

0

Others

$1(1.0 \%)$

$1(1.0 \%)$

0

Type of operation

$\begin{array}{lllll}\text { LAR } & 80(76.9 \%) & 78(77.2 \%) & 2(66.7 \%) & 0.212 \\ \text { AR } & 10(9.6 \%) & 10(9.9 \%) & 0 & \\ \text { TC } & 6(5.8 \%) & 6(5.9 \%) & 0 & \\ \text { TPC } & 5(4.8 \%) & 4(4.0 \%) & 1(33.3 \%) & 0.282 \\ \text { RHC } & 3(2.9 \%) & 3(3.0 \%) & 0 & \\ \text { Associated bowel resection } & 79(76.0 \%) & 77(76.2 \%) & 2(66.7 \%) & \\ \text { No } & 7(6.7 \%) & 6(5.9 \%) & 1(33.3 \%) & \\ \text { Colectomy } & 8(7.7 \%) & 8(7.9 \%) & 0 & \\ \text { Small intestine resection } & 10(9.6 \%) & 10(9.9 \%) & 0 & \\ \text { Appendectomy } & & & \\ \text { Level of anastomosis from AV } & 15(14.4 \%) & 13(12.9 \%) & 2(66.7 \%) \\ \text { (cm) } & 63(60.6 \%) & 62(61.4 \%) & 1(33.3 \%) \\ <5 & 6(5.8 \%) & 6(5.9 \%) & 0 & \\ 5 \sim 8 & 20(19.2 \%) & 20(19.8 \%) & 0\end{array}$

No data 


\section{Total $(n=104)$ \\ No leakage $(\mathrm{n}=$ \\ 101) \\ With leakage (n \\ $=3$ ) \\ P- value}

\section{FIGO stage}

(1)

II

Ila

4 (3.8\%)

$4(4.0 \%)$

0

0.991

Ilb

IIC

III

IIIa

13 (12.5\%)

$13(12.9 \%)$

0

$3(2.9 \%)$

$3(3.0 \%)$

0

6 (5.8\%)

$6(5.9 \%)$

0

4 (3.8\%)

$4(4.0 \%)$

0

48 (46.1\%)

47 (46.6\%)

$1(33.3 \%)$

Illa

$2(1.9 \%)$

$2(2.0 \%)$

0

IIIb

$2(1.9 \%)$

$2(2.0 \%)$

0

IIIC

44 (42.3\%)

$43(42.6 \%)$

1 (33.3\%)

IV

36 (34.6\%)

34 (33.3\%)

$2(66.7 \%)$

$\mathrm{IVa}$

$13(12.5 \%)$

12 (11.9\%)

$1(33.3 \%)$

IVb

$23(22.1 \%)$

$22(21.8 \%)$

1 (33.3\%)

No data

3 (2.9\%)

3 (2.9\%)

0

Recurrence

\begin{tabular}{lllll|} 
First diagnosis & $92(88.5 \%)$ & $89(88.1 \%)$ & $3(100 \%)$ & 0.690 \\
Recurrence & $12(11.5 \%)$ & $12(11.9 \%)$ & 0 & \\
Anastomosis & & & & \\
Stapler & $102(98.1 \%)$ & $99(98.0 \%)$ & $3(100.0 \%)$ & 0.943 \\
Hand-sewn & $2(1.9 \%)$ & $2(2.0 \%)$ & 0 & \\
Preop chemotherapy & & & $3(100.0 \%)$ & 0.905 \\
No & $88(84.6 \%)$ & $85(84.2 \%)$ & 0 & \\
Preop neoadjuvant & $4(3.8 \%)$ & $4(4.0 \%)$ & 0 & \\
Preop CCRT & $3(2.9 \%)$ & $3(3.0 \%)$ & 0 & \\
Postop adjuvant & $9(8.7 \%)$ & $9(8.9 \%)$ & &
\end{tabular}




\begin{tabular}{|c|c|c|c|c|}
\hline & Total $(n=104)$ & $\begin{array}{l}\text { No leakage }(n= \\
101)\end{array}$ & $\begin{array}{l}\text { With leakage ( } \mathrm{n} \\
=3 \text { ) }\end{array}$ & $\begin{array}{l}\mathrm{P} \text { - } \\
\text { value }\end{array}$ \\
\hline \multicolumn{5}{|l|}{ Preop radiotherapy } \\
\hline No & $100(96.2 \%)$ & $97(96.0 \%)$ & $3(100 \%)$ & 0.940 \\
\hline Preop CCRT & $3(2.9 \%)$ & $3(3.0 \%)$ & 0 & \\
\hline Preop radiotherapy only & $1(1.0 \%)$ & $1(1.0 \%)$ & 0 & \\
\hline \multicolumn{5}{|l|}{ Tumor marker } \\
\hline CA125 & (98) & (95) & (3) & 0.697 \\
\hline Normal $(<36)$ & $11(11.2 \%)$ & $11(11.6 \%)$ & 0 & 0.396 \\
\hline Abnormal & $87(88.8 \%)$ & $84(88.4 \%)$ & $3(100 \%)$ & - \\
\hline CA19-9 & (79) & $(76)$ & (3) & \\
\hline Normal $(<36)$ & $65(62.5 \%)$ & $62(61.4 \%)$ & $3(100 \%)$ & \\
\hline Abnormal & $14(13.5 \%)$ & $14(13.9 \%)$ & 0 & \\
\hline SCC & (7) & (7) & $(0)$ & \\
\hline Normal $(<=1.0)$ & $1(14.3 \%)$ & $1(14.3 \%)$ & & \\
\hline Abnormal $(>1.0)$ & $4(57.1 \%)$ & $4(57.1 \%)$ & & \\
\hline No data & $2(28.6 \%)$ & $2(28.6 \%)$ & & \\
\hline
\end{tabular}

\section{Discussion}

Since gynecological malignancies, including ovarian cancer, frequently invade adjacent organs in the pelvis at advanced stages, lower colonic and rectal resections are often combined for complete resection of the lesion [3, 4]. In the case of ovarian cancer, it was reported that colorectal resection was performed concurrently in about $26 \%$ of patients who underwent cytoreductive operations for complete resection of the tumor [8]. In tumor reduction surgery for ovarian cancer, colectomy occupies an important position due to frequent invasions into the colon (about $50 \%$ or more in some previous studies), technical problems, and postoperative complications. In many studies, colectomy for complete tumor reduction was found to have a positive effect on survival rate $[8,9]$.

The most commonly invaded parts of the gastrointestinal tract were the sigmoid colon and the rectum [3, $8,10]$, followed by the ileoceacum [8]. In this study as well, LAR, which is rectal resection, showed the highest frequency at $76.9 \%$ followed by AR at $9.6 \%$, which were confirmed to be similar to previous studies.

There was no case of death as a direct result of postoperative complications among the subjects of this study. One patient died of multi-organ failure one month after the operation. However, she was old at the 
time of diagnosis, and it was judged that the multi-organ failure had progressed due to the rapid progression of the cancer as the cancer was at FIGO stage IVb. The frequencies of postoperative complications by type were gastrointestinal system complications (17.3\%), urinary system complications (15.4\%), wound infection (7.7\%), cardiovascular system complications (6.7\%), and intraabdominal infection (excluding anastomotic site-related ones) (5.8\%). Given the surgical sites and anatomical positions, the relatively higher frequencies of gastrointestinal and urinary system complications can be regarded to be reasonable.

Anastomotic leakage is one of the most fatal complications after colorectal resection. It is diagnosed by checking intestinal contents such as digestive fluid coming out of the drainage tube or fluid retention in the abdominal cavity or abdominal wall. It has been reported in $2.8 \sim 23 \%$ of general colorectal surgery and in $0.8 \sim 6.8 \%$ of gynecological malignancy surgery $[1,4]$. Commonly known major risk factors for anastomotic leakage include cases where the anastomotic site is located under the peritoneal reflection, preoperative radiotherapy, advanced stage, emergency surgery, blood transfusion, malnutrition, obesity, smoking, steroid therapy, and automatic stapler-related factors [4-6]. In this study, the frequency of anastomotic leakage that occurred was three patients (2.9\%) out of the 104 patients, which was lower compared to the frequency of anastomotic leakage after colon and rectal cancer surgery $(2.8 \sim 23 \%)$ mentioned earlier. It was shown to be similar to the frequency of anastomotic leakage after colorectal resection for primary colorectal cancer (81 out of 2391 patients, 3.4\%) performed at the same research institute during the same period (December 2008 to August 2020) and was similar to that of the primary colorectal cancer patients.

According to some previous studies, the reason why the frequency of anastomotic leakage in collaborated coloproctectomies in gynecological malignancies is lower than in coloproctectomies in primary colon cancer is that the blood flow and tissues of the intestine in the case of gynecological malignancies are relatively stable compared to those in the case of primary colon cancer because the invasion of lesions is mostly confined to the superficial layers of the colon and the rectum, and anastomotic sites are much stable because they are located at relatively higher positions from the anal verge $[3,11,12]$. The low frequency of anastomotic leakage in this study is also thought to be due to these characteristics of gynecological malignancies.

Among the risk factors for anastomotic leakage analyzed in this study, the preoperative albumin level was the only variable of which the statistical significance was identified. In some previous studies, it was identified that the risk of anastomotic leakage increased when the albumin level was low $[4,6]$. Since albumin is a serum protein, which is an indicator of the patient's nutritional status, delay in the anastomotic site wound recovery process due to malnutrition seems to increase the risk of leakage [6]. However, there were also studies where there was no significant association between albumin levels and anastomotic leakage [5], and some studies mentioned that it was difficult to accurately analyze albumin levels as a risk factor because a considerable amount of preoperative albumin level data were missing [4, 5]. In this study, since albumin was included in the basic preoperative examination items of all subject 
patients, the correlation between preoperative albumin levels and anastomotic leakage could be identified.

This study has a limitation that it is a single-center retrospective study with a small number of samples. In addition, since the frequency of occurrence of anastomotic leakage was extremely low, there was no statistical significance in the comparison of risk factors in most cases. However, since the findings of this study are data from a comprehensive investigation and analysis of cases of collaborative colorectal resection among gynecological malignancy patients treated at the study target institution for 12 years since the opening of the institution, the data are thought to be valuable as preceding research data when studies are conducted later with more patients.

\section{Conclusion}

When colonic and rectal metastasis have been confirmed in a gynecological malignancy patient, colorectal resection can be performed simultaneously. Complications accompanying intestinal resection, especially fatal complications such as anastomotic leakage, may occur, but the frequency is extremely low. Therefore, radical resection can be actively performed in consideration of the patient's overall conditions and risk factors.

\section{Abbreviations}

Alb, albumin; AR, anterior resection; ASA, American Society of Anesthesiology; AV, anal verge; BMI, body mass index; CCRT, concurrent chemoradation therapy; DM, diabetes mellitus; EBL, estimated blood loss; FFP, fresh frozen plasma; FIGO, International Federation of Gynecology and Obstetrics; $\mathrm{Hb}$, hemoglobin; LAR, low anterior resection; RBC, red blood cell; RHC, right hemicolectomy; SCC, squamous cell carcinoma antigen, TC, total colectomy; TPC, total proctocolectomy

\section{Declarations}

\section{Ethics approval and consent to participate}

This study protocol was approved by the Institutional Review Board (IRB) of Pusan National University Yangsan Hospital. (IRB No. 05-2020-203). Written informed consent forms concerning cooperative colorectal resection were obtained for publication of the study.

\section{Consent for publication}

We obtained written consent to publish all the personal details included in our dataset from all participants prior to surgery.

\section{Availability of data and materials}

All datasets on which the conclusions of the manuscript rely was provided as additional supporting file. 


\section{Competing interests}

The authors declare that they have no competing interest.

\section{Funding}

The authors declare that no funding was received for the study.

\section{Authors' contributions}

HSK designed the study, analyzed and interpreted the data, and made a draft and revised the manuscript. AK analyzed and interpreted data and wrote the manuscript. BP, SHC helped to acquire the data and performed the statistical analysis. KMS, YJS was responsible for the study's conception and design. All authors have read and approved the final manuscript.

\section{Acknowledgements}

We would like to thank our colleagues and nurses who took care of the patients.

\section{References}

1. Kalogera E, Nitschmann CC, Dowdy SC, Cliby WA, Langstraat CL. A prospective algorithm to reduce anastomotic leaks after rectosigmoid resection for gynecologic malignancies. Gynecol Oncol. 2017;144(2):343-7.

2. Lago V, Fotopoulou C, Chiantera V, Minig L, Gil-Moreno A, Cascales-Campos PA, et al. Risk factors for anastomotic leakage after colorectal resection in ovarian cancer surgery: A multi-centre study. Gynecol Oncol. 2019;153(3):549-54.

3. Son JH, Kim J, Shim J, Kong TW, Paek J, Chang SJ, et al. Comparison of posterior rectal dissection techniques during rectosigmoid colon resection as part of cytoreductive surgery in patients with epithelial ovarian cancer: Close rectal dissection versus total mesorectal excision. Gynecol Oncol. 2019;153(2):362-7.

4. Richardson DL, Mariani A, Cliby WA. Risk factors for anastomotic leak after recto-sigmoid resection for ovarian cancer. Gynecol Oncol. 2006;103(2):667-72.

5. Kalogera E, Dowdy SC, Mariani A, Weaver AL, Aletti G, Bakkum-Gamez JN, et al. Multiple large bowel resections: potential risk factor for anastomotic leak. Gynecol Oncol. 2013;130(1):213-8.

6. Lago V, Fotopoulou C, Chiantera V, Minig L, Gil-Moreno A, Cascales-Campos PA, et al. Risk factors for anastomotic leakage after colorectal resection in ovarian cancer surgery: A multi-centre study. Gynecol Oncol. 2019.

7. Grimm C, Harter P, Alesina PF, Prader S, Schneider S, Ataseven B, et al. The impact of type and number of bowel resections on anastomotic leakage risk in advanced ovarian cancer surgery. Gynecol Oncol. 2017;146(3):498-503. 
8. Hoffman MS, Zervose E. Colon resection for ovarian cancer: intraoperative decisions. Gynecol Oncol. 2008;111(2 Suppl):S56-65.

9. Plotti F, Montera R, Aloisi A, Scaletta G, Capriglione S, Luvero D, et al. Total rectosigmoidectomy versus partial rectal resection in primary debulking surgery for advanced ovarian cancer. Eur J Surg Oncol. 2016;42(3):383-90.

10. Kebapci E, Gulseren V, Tugmen C, Gokcu M, Solmaz U, Sert I, et al. Outcomes of patients with advanced stage ovarian cancer with intestinal metastasis. Ginekol Pol. 2017;88(10):537-42.

11. Mourton SM, Temple LK, Abu-Rustum NR, Gemignani ML, Sonoda Y, Bochner BH, et al. Morbidity of rectosigmoid resection and primary anastomosis in patients undergoing primary cytoreductive surgery for advanced epithelial ovarian cancer. Gynecol Oncol. 2005;99(3):608-14.

12. Fotopoulou C, Jones BP, Savvatis K, Campbell J, Kyrgiou M, Farthing A, et al. Maximal effort cytoreductive surgery for disseminated ovarian cancer in a UK setting: challenges and possibilities. Arch Gynecol Obstet. 2016;294(3):607-14.

\section{Figures}

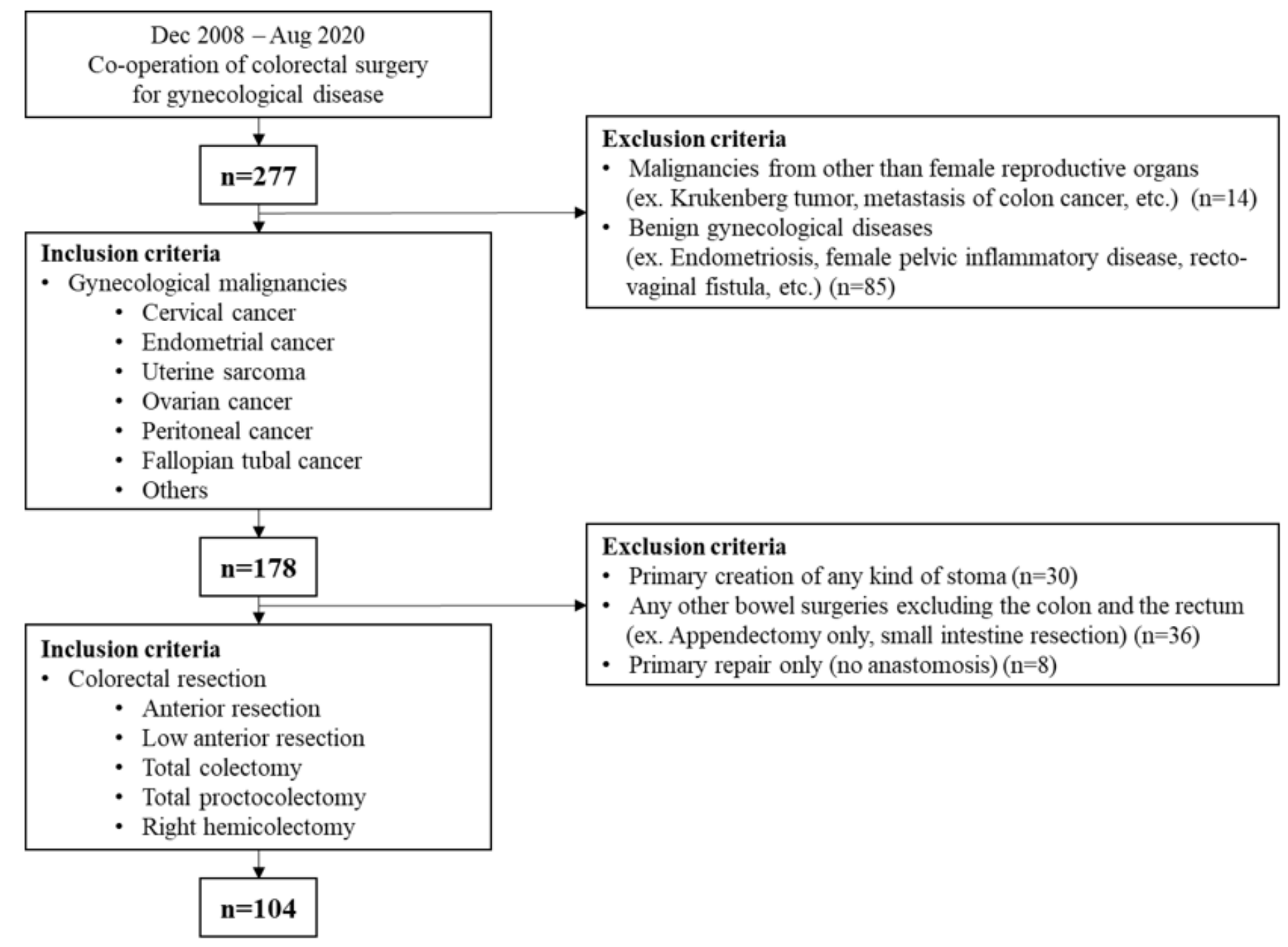


Figure 1

Flowcharts for inclusion and exclusion 